\title{
Cosmological distances and fractal statistics of galaxy distribution
}

\begin{abstract}
M. B. Ribeiro
Physics Institute, University of Brazil-UFRJ, CxP 68532, CEP 21945-970, Rio de Janeiro, Brazil e-mail: mbr@if.ufrj.br

Received 15 June 2004 / Accepted 6 August 2004

Abstract. This paper studies the effect of the distance choice in radial (non-average) statistical tools used for fractal characterization of galaxy distribution. After reviewing the basics of measuring distances of cosmological sources, various distance definitions are used to calculate the differential density $\gamma$ and the integral differential density $\gamma^{*}$ of the dust distribution in the Einstein-de Sitter cosmology. The main results are as follows: (1) the choice of distance plays a crucial role in determining the scale where relativistic corrections must be taken into account, as both $\gamma$ and $\gamma^{*}$ are strongly affected by such a choice; (2) inappropriate distance choices may lead to failure to find evidence of a galaxy fractal structure when one calculates those quantities, even if such a structure does occur in the galaxy distribution; (3) the comoving distance and the distance given by Mattig's formula are unsuitable to probe for a possible fractal pattern as they render $\gamma$ and $\gamma^{*}$ constant for all redshifts; (4) a possible galaxy fractal system at scales larger than $100 \mathrm{Mpc}(z \approx 0.03)$ may only be found if those statistics are calculated with the luminosity or redshift distances, as they are the ones where $\gamma$ and $\gamma^{*}$ decrease at higher redshifts; (5) Célérier \& Thieberger's (2001) critique of Ribeiro's (1995) earlier study are rendered impaired as their objections were based on misconceptions regarding relativistic distance definitions.
\end{abstract}

Key words. cosmology: theory - cosmology: large-scale structure of the Universe - cosmology: observation galaxies: distances and redshifts - galaxies: statistics

\section{Introduction}

From the very beginnings of modern cosmology the question exists of whether or not there will be a distance scale where the matter distribution in the Universe becomes uniformly distributed, or if it will always remain inhomogeneous, following, in this case, a hierarchical clumping pattern of matter. With the adoption of the Cosmological Principle by the majority of researchers working in cosmology, it became standard to equate the former hypothesis to a statement of truth, meaning that most researchers adopted the view that there must be a scale beyond which a homogeneous distribution will be reached. This is the viewpoint presented in most, if not all, textbooks in cosmology. As a consequence the latter hypothesis, that is, the alternative hierarchical, or inhomogeneous, viewpoint was pushed to the sidelines. Nevertheless, and despite the historical fact that since the beginning of modern cosmology, in the 1920s and 1930s, the view based on the Cosmological Principle became dominant, the hierarchical proposal managed to survive on the fringes of cosmological research for almost a century (de Vaucouleurs 1970; Mandelbrot 1977, 1983; Pietronero 1987; Ribeiro 1994).

The modern version of a hierarchical distribution of matter in the Universe is due to Charlier $(1908,1922)$, who followed earlier concepts from Fournier d'Albe (1907). Nevertheless, the hierarchical matter structuring concept is by no means new, but goes to as far back as the pressocratic Greek philosopher Anaxagoras (Grujić 2001), and having in its list of defenders in the last 450 years thinkers like Giordano Bruno, Kant, Leibniz (who opposed Newton's homogeneous universe view) and Laplace, among others (see Grujić 2001, 2002; and Baryshev \& Teerikorpi 2002, for historical accounts of those pre-modern hierarchical ideas). More recently, however, the dispute between these two views took an interesting new turn when Mandelbrot $(1977,1983)$ and Pietronero (1987) proposed that the old hierarchical concept is nothing more than the assumption that the matter in the Universe should be distributed according to a fractal pattern ${ }^{1}$.

An important technical aspect of this dispute is that during most of its modern century-old history, hierarchical modelling remained almost exclusively confined to the realm of Newtonian cosmology (Charlier 1922; Wertz 1970, 1971;

1 Wertz $(1970,1971)$ had reached similar conclusions much earlier when he advanced a model mathematically identical to Pietronero's. Although he never used the word "fractal", Wertz's treatment did assume a scale-invariant, or self-similar, pattern for the galaxy distribution (see Ribeiro 1994; and Ribeiro \& Miguelote 1998, for comparisons between these two models). Unfortunately his model remained virtually unknown for decades, even to Mandelbrot $(1977,1983)$ and Pietronero (1987). 
Haggerty \& Wertz 1972; Mandelbrot 1977, 1983; Pietronero 1987; Coleman \& Pietronero 1992; Sylos-Labini et al. 1998; Ribeiro \& Miguelote 1998; Abdalla et al. 1999; Pietronero \& Sylos-Labini 2000; Pietronero et al. 2003). Although 20th century scientific cosmology was basically relativistic cosmology since its establishment in 1917 by Einstein, attempts to model the hierarchical concept within a relativistic framework had to wait until the 1970s when the first tentative relativistic models were advanced (Bonnor 1972; Wesson 1978, 1979)².

In the early 1990s, this author took a fresh view of the relativistic side of this issue, and concluded that when a fully relativistic model is considered the problem of observationally identifying whether or not a uniform distribution of matter is really being observed is much less straightforward than previously assumed (Ribeiro 1992a,b, 1993, 1994). Following this line of reasoning to its logical consequences, Ribeiro (1995) concluded that in an Einstein-de Sitter (EdS) cosmology the spatial homogeneity of this model may only be observed at much closer ranges than previously assumed, up to $z \approx 0.01$, which led this author to conjecture that the observed fractal structure may be an observational effect of geometrical nature due to the fact that astronomical observations are always carried out along our past null cone. This means that the fractal, or hierarchical, hypothesis for the matter distribution in the Universe may not be in contradiction with the Cosmological Principle (Ribeiro 2001a,b).

The conclusions reached in Ribeiro (1995) regarding the observable depth of the homogeneous region in the EdS model were, however, challenged by Célérier \& Thieberger (2001) who claim that, contrary to Ribeiro (1995), spacetime curvature will only show its effects at redshift depths much larger than the range calculated in Ribeiro (1995).

The aim of this paper is to discuss further the issues behind the relativistic side of this dispute, as well as providing a reply to Célérier \& Thieberger (2001). This article also presents results which extend and complement both Ribeiro (1995 and 2001b). In what follows it is shown that the radial (non-average) statistical tools used in fractal analyses of the galaxy distribution, namely the differential density $\gamma$ and the integral differential density $\gamma^{*}$, depend strongly on the choice of observational distance. Taking the EdS cosmology as the model of choice due to its simplicity, it is possible to define four different observational distances, namely the area distance $d_{\mathrm{A}}$, galaxy area distance $d_{\mathrm{G}}$, redshift distance $d_{z}$ and luminosity distance $d_{L}$. Proper and comoving distances are also defined, but the former is equal to $d_{\mathrm{A}}$ whereas the latter is equal to $d_{\mathrm{G}}$ times a constant factor. The distance given by Mattig's formula is also used, but it turns out to be the same as $d_{\mathrm{G}}$. In addition, it is shown that in view of the fact that some of these observational distances go asymptotically to infinity as one approaches the big bang whereas others tend to zero, the above mentioned statistical functions are strongly affected. In particular, the $\gamma$ statistic defined with the area distance diverges at $z=1.25$, losing then its analytical usefulness for higher redshifts.

\footnotetext{
${ }^{2}$ Earlier discussions on the relativistic side of this dispute were reported by Wertz (1970; see also Ribeiro 1994).
}

The study presented here also suggests that the galaxy area distance, the comoving distance and, still, the distance given by Mattig's formula are unsuitable to probe for a fractal pattern as they render both $\gamma$ and $\gamma^{*}$ constant for all redshift. Therefore, if a fractal pattern really exists at ranges greater than $100 \mathrm{Mpc}$, which is the scale where both $\gamma$ and $\gamma^{*}$ diverge depending on the choice of distance, this pattern could only be found at that scale if one carries out statistical analyses with either the redshift or luminosity distances, as these are the only distance definitions capable of leading $\gamma$ and $\gamma^{*}$ to a decrease at higher redshifts. Those findings confirm and complement similar results obtained earlier by Ribeiro (1992b, 1995, 2001b).

The dependence of the radial (non-average) statistical functions on the choice of distance can be seen if we remember that any galaxy statistic requires us to define the area of a spherical shell of a certain radius and since we have more than one distance definition we will inevitably end up with various expressions for such shell areas, none of them being better than the others, a conclusion opposite to Célérier \& Thieberger (2001). Actually, their criticism confuses observables with the relationships that relate them to one another and the underlying cosmological model. Clarifying this point renders incorrect both their calculations and interpretation. The main misconception is about relativistic distances and how they are defined and used. Inasmuch as this issue, cosmological distances, still causes confusion, and is at the heart of this and related issues (Ribeiro 2001b), further discussions of this subject is justified. If the meaning and use of cosmological distances are clarified, perhaps future errors of this sort will be avoided.

The plan of the paper is as follows. Section 2 reviews the issue of measuring distances of cosmological sources. Section 3 develops radial $\gamma$ type dust statistics in the EdS cosmology, and Sect. 4 analyzes the results and suggests that observers should consider probing for a possible fractal pattern in the galaxy distribution by using average statistics with the various distance definitions used in this paper in order to ascertain whether or not distortions due to the choice of distance are also present in the final results. The paper ends with a concluding section.

\section{Measuring distances of cosmological sources}

In cosmology, observational distances are defined by the method of measurement. This means that once we collect some astronomical data of cosmological relevance, say, for instance, redshift of distant galaxies, their apparent magnitudes, some intrinsic source size, etc, the particular way we relate one observational quantity to another defines a particular observational distance. In other words, in cosmology, measuring a distance depends on circumstances. In a flat and Euclidean space all methods of measurement lead to the same and unique distance. In relativistic cosmology, however, that is not the case and we are then forced to give up the notion of a correct, or right, distance. In relativity, distance is not an absolute concept.

The lack of a unique distance notion means that other quantities used in cosmology where distance appears in their definitions must not be unique either. This is the case of volume, spherical shell, average density, and, of course, any statistic of galaxy distribution since they require distance-derived 
quantities. However, as in our neighbourhood we can take a Newtonian cosmology approximation where all relativistic distances become the same, a relevant question then arises as to where those quantities will start to deviate from these Newtonian values. This question is far from trivial as general relativity is a non-linear gravitational theory and this means that each observational quantity may have its own particular deviation regime, and which may substantially differ from other ones (Ribeiro 2001b).

Observational distance definitions in cosmology are an issue that has been already well established in relativistic cosmology for over thirty years since Ellis' (1971) seminal contribution to this subject. In observational cosmology, however, the generality of Ellis' conclusions do not yet seem to have been fully appreciated. For instance, Kayser et al. (1997) dealt with cosmological distances, as did Hogg (1999), who collected the various definitions in use in the astronomical literature. Nevertheless, neither quoted Ellis' (1971) fundamental work on this matter, nor did they seem to have appreciated Ellis' results that showed how to calculate cosmological distances for any cosmological spacetime metric. Actually, the roots of this discussion go back as far as to the famous Etherington reciprocity theorem, proved in 1933, and rediscovered by R. Penrose and R. K. Sachs in the 1960s (Ellis 1971, p. 111; see also Schneider et al. 1992).

An observer, of course, may wisely ignore this whole discussion if one is only interested in relating observational quantities to other observational quantities. In this approach, which may be called "Sandage's prescription" (see Ribeiro 2001b), distances become an internal parameter of the theory and the choice of distance turns out to be irrelevant. The limitation of this approach is that if the final quantities are explicitly dependent on distances, which is the case for the average density and many statistical tools of galaxy distribution, Sandage's prescription is inapplicable.

Having said all above, it is therefore wise to approach the subject theme of this paper very carefully. Thus, I start by describing the various observational distances appearing in Ellis' (1971) paper, and then proceed to the theoretical issues.

\subsection{Observational distances}

The luminosity distance $d_{L}$ is defined if one assumes a flat and non-expanding universe, as if it were Euclidean, and relates apparent magnitude, or observed flux $F$, with the intrinsic luminosity $L$ of the source by means of the well-known expression

$$
F=\frac{L}{4 \pi\left(d_{L}\right)^{2}}
$$

The area distance $d_{\mathrm{A}}$ (also known as angular diameter distance, corrected luminosity distance or observer area distance $)^{3}$ is measured by means of the relationship between an

\footnotetext{
${ }^{3}$ In this paper I will adopt Ellis' (1971) terminology for observational distances, as I believe his name choices to be the least confusing, and, geometrically, most appropriate.
}

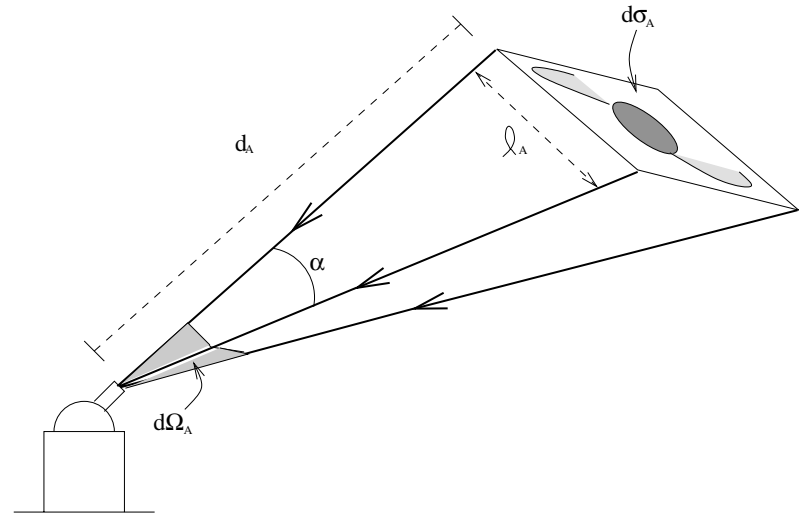

Fig. 1. The area distance $d_{\mathrm{A}}$ is obtained by the relationship between the intrinsic cross-sectional area $\mathrm{d} \sigma_{\mathrm{A}}$ of the source, measured at its restframe, and the solid angle $\mathrm{d} \Omega_{\mathrm{A}}$ measured by the observer (Eq. (2)). If an intrinsic dimension $\ell_{\mathrm{A}}$ can be measured at the source's rest-frame and its corresponding angular dimension $\alpha$ is measured by the observer, one can estimate $d_{\mathrm{A}}$ (Eq. (5)). In the Einstein-de Sitter cosmology $d_{\mathrm{A}}$ is the same as the proper distance (see Sect. 3.3).

intrinsically measured cross-sectional area element $\mathrm{d} \sigma_{\mathrm{A}}$ of the source and the observed solid angle $\mathrm{d} \Omega_{\mathrm{A}}$ (see Fig. 1),

$\mathrm{d} \sigma_{\mathrm{A}}=\mathrm{d} \Omega_{\mathrm{A}}\left(d_{\mathrm{A}}\right)^{2}$.

The galaxy area distance $d_{\mathrm{G}}$ (also known as effective distance, angular size distance, transverse comoving distance or proper motion distance) is similar, but defined from the opposite point where the area distance is specified. The solid angle $d \Omega_{G}$ is measured at the rest frame of the galaxy, whereas the intrinsic cross-sectional area $\mathrm{d} \sigma_{\mathrm{G}}$ is measured here (see Fig. 2). The defining equation may be written as,

$\mathrm{d} \sigma_{\mathrm{G}}=\mathrm{d} \Omega_{\mathrm{G}}\left(d_{\mathrm{G}}\right)^{2}$.

The methods above for relating observables define distances that are in principle directly measurable and do not necessarily need to coincide ${ }^{4}$. Besides $d_{L}, d_{\mathrm{A}}, d_{\mathrm{G}}$, the equations above also define three quantities which are measured here, that is, at the observer's location, namely $F, \mathrm{~d} \Omega_{\mathrm{A}}, \mathrm{d} \sigma_{\mathrm{G}}$, and, additionally, three other quantities which can only be measured at the source's rest frame, namely $L, \mathrm{~d} \sigma_{\mathrm{A}}, \mathrm{d} \Omega_{\mathrm{G}}$.

Notice that as $d_{L}$ and $d_{\mathrm{A}}$ are directly determinable quantities, without assuming any cosmological model, their measurements are, therefore, model independent. In fact, precise determination of these quantities can be used to distinguish among cosmological models. $d_{\mathrm{G}}$ is not directly measurable since $\mathrm{d} \Omega_{\mathrm{G}}$ cannot be determined from the observer's location. To be useful, $d_{\mathrm{G}}$ requires a further equation relating it to $d_{L}$ or $d_{\mathrm{A}}$ (see Sect. 2.2 below).

\subsection{Etherington's reciprocity theorem}

The observational distances discussed above are connected to one another and the redshift $z$ by a very important theorem

\footnotetext{
${ }^{4}$ By means of galactic parallax one can still define and measure a fourth distance, the parallax distance $d_{\mathrm{P}}$ (Ellis 1971). However, we are still unable to measure galactic parallaxes and, therefore, $d_{\mathrm{P}}$ is of no use in our discussion here.
} 


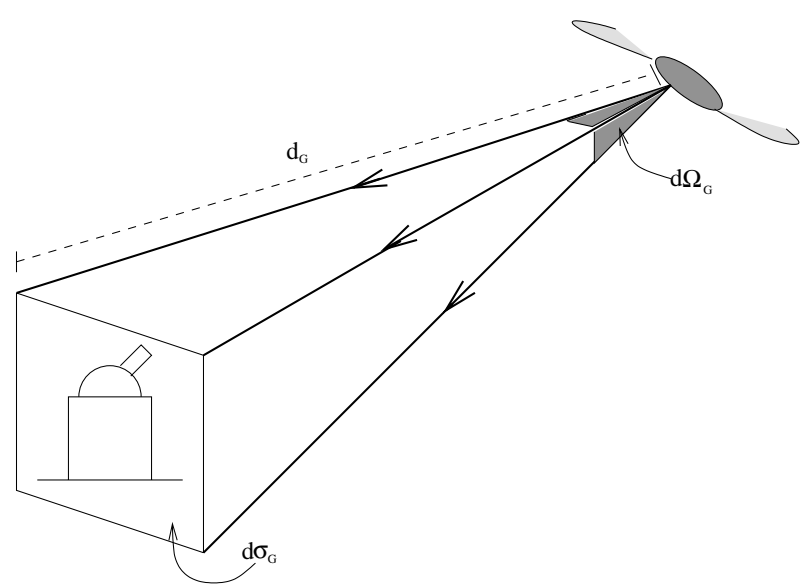

Fig. 2. The galaxy area distance $d_{\mathrm{G}}$ is obtained by the relationship between the cross-sectional area $\mathrm{d} \sigma_{\mathrm{G}}$ measured at the observer's restframe, and the solid angle $\mathrm{d} \Omega_{\mathrm{G}}$ measured at the source's rest-frame (Eq. (3)). In the Einstein-de Sitter cosmological model this is the same distance as given by Mattig's formula, also having the same behaviour against $z$ as the comoving distance (see Sect. 3.3).

proved a long time ago by Etherington (1933), called the reciprocity theorem or Etherington's reciprocity law. It may be written as (Ellis 1971; Schneider et al. 1992, pp. 111, 116),

$d_{L}=(1+z)^{2} d_{\mathrm{A}}=(1+z) d_{\mathrm{G}}$,

and it is valid for any cosmological model. In fact the theorem is purely geometrical and only requires that source and observer are connected by null geodesics. It is simply a consequence of the geodesic deviation equation and it tells us that if $z=0$ that implies that equal surface elements $\mathrm{d} \sigma_{\mathrm{G}}, \mathrm{d} \sigma_{\mathrm{A}}$ subtend equal solid angles $\mathrm{d} \Omega_{\mathrm{G}}, \mathrm{d} \Omega_{\mathrm{A}}$, irrespective of the curvature of spacetime (Ellis 1971). As this theorem is valid for all cosmologies, it has been recently suggested that it can be a powerful tool for testing non-standard cosmological models, as well as being capable of distinguishing between various models of dark energy by comparing distances measured by standard candles, that is, $d_{L}$, and standard intrinsic dimensions, i.e., $d_{\mathrm{A}}$ (Basset \& Kunz 2004; Uzan et al. 2004).

\subsection{The necessity of a cosmological model}

So far we have not needed to establish any cosmological model, but as we have five expressions (Eqs. (1)-(4)) containing four quantities measurable here, $F, \mathrm{~d} \Omega_{\mathrm{A}}, \mathrm{d} \sigma_{\mathrm{G}}, z$, plus six unknown quantities, $L, d_{L}, \mathrm{~d} \sigma_{\mathrm{A}}, d_{\mathrm{A}}, \mathrm{d} \Omega_{\mathrm{G}}, d_{\mathrm{G}}$, we end up with an unsolvable system. However, if we are able to independently determine either $L$ or $\mathrm{d} \sigma_{\mathrm{A}}$, the system can be solved. In both cases we require knowledge of the intrinsic physics of the source, or source evolution, a theory which is still lacking for galaxies.

There is a possible way to solve the system by determining $d_{\mathrm{A}}$. Let us call $\alpha$ the observed angular dimension of some object whose linear intrinsic dimension $\ell_{\mathrm{A}}$, perpendicular to the line of sight can be estimated (see Fig. 1). Then,

$\ell_{\mathrm{A}} \approx d_{\mathrm{A}} \alpha$

and we can determine the area distance to a reasonable degree of accuracy. Notice, however, that $\ell_{\mathrm{A}}$ is measured at the source's rest frame, which means that we again need knowledge of the intrinsic physics of the source in order to estimate $\ell_{\mathrm{A}}$ and then calculate $d_{\mathrm{A}}$. This remark will be very important in what follows.

Another attempt at looking at the difficulties above was the ideal observational cosmology program, which proposed to characterize in detail the way in which cosmological observations can be directly used to determine the cosmological spacetime geometry (Ellis et al. 1985). The main motivation behind this program is to determine the spacetime metric of our universe directly from astronomical observations, without assuming a cosmological model beforehand. In the process of doing that, one must first determine what is and what is not decidable in cosmology on the basis of astronomical observations. Although this is an appealing idea, this program cannot be fully implemented due to the astrophysical evolution, both in number and in luminosity, of galaxies. To implement this program we require precise observations of $d_{L}$ or $d_{\mathrm{A}}$, redshift, galaxy number counts and an adequate model of how the populations and luminosities of galaxies of different morphologies evolve with $z$, that is, to know independently the function $L=L(z)$ for each morphological galaxy type. It is presently impossible to obtain all that information without assuming a cosmological model.

Another way of possibly solving this system of equations and determining all quantities described so far is to find a homogeneous class of sources, that is, objects which share some basic features, and whose intrinsic luminosity $L$ may be known. This is, of course, the idea behind supernova cosmology. The limitations of this approach are that the measured intrinsic supernova Ia luminosities at high $z$ assume no luminosity evolution at different redshifts due to limitations in our theoretical understanding of these objects (Perlmutter \& Schmidt 2003; Li $\&$ Filippenko 2003). In addition, the building blocks of the universe are galaxies and not supernovas, and the picture emerging from supernova cosmology ought to be compatible with galactic physics and observations. Nevertheless, this is currently a very promising line of research in observational cosmology.

So, unless supernova cosmology, or another observational methodology, firmly establishes cosmological modelindependent relationships between values of $d_{L}$ and $d_{\mathrm{A}}$ at different $z$, we have no other way but to assume an universe model in order to obtain another equation, usually in the functional form of either $d_{L}(z)$, or $d_{\mathrm{A}}(z)$, or $d_{\mathrm{G}}(z)$, and be able to fully solve the system of equations above.

\subsection{Theoretical distances}

Once a cosmological model is assumed, we can, of course, define other, model dependent, distances like the comoving distance, proper distance, interval distance, geodesic distance, absolute distance, etc. These are all, however, different forms of line element separations $\left(\mathrm{d} s^{2}\right)$, whose theoretically-defined expressions are entirely dependent on the spacetime geometry and the particular solution of Einstein's field equations ${ }^{5}$.

\footnotetext{
5 Notice that the reciprocity theorem does not require a solution of Einstein's field equations, but only the pseudo-Riemannian spacetime.
} 
Their particular expressions change according to the cosmological model and, hence, they are not observationally defined distances, although they do play an important theoretical role in cosmology.

\subsection{The consistency problem in observational cosmology}

Since most observational results in cosmology either explicitly or implicitly assume a cosmological model, usually the standard one, in their presentation, a question arises immediately. Do the observational results really determine parameters compatible with the assumed cosmological model, as they should? This is the consistency problem in observational cosmology (Ribeiro \& Stoeger 2003). Consistency tests are, therefore, desirable, in order to check the agreement between the derived observational parameters and the cosmological model they assume. This means that we can only discuss the possible departures from a homogeneous matter distribution if we choose an average density that theoretically allows such a departure and then check the model for consistency with the data. In order words, once we calculate observational parameters by means of a cosmological model, the agreement or disagreement between the data and the model will be tested by consistency or inconsistency.

\section{Radial dust statistics in the Einstein-de Sitter cosmology}

Let us define another relation which may be called an observational distance, the redshift distance $d_{z}$. It may be written as,

$d_{z}=\frac{c z}{H_{0}}$,

where, $c$ is the light speed and $H_{0}$ is the Hubble constant. This is, of course, just a consequence of the velocity-distance equation and the Doppler approximation in a expanding universe, being valid only for $z<1$ (Harrison 1993). It is not an observational distance in the sense as discussed above, but a quantity proportional to small redshifts in the standard cosmology. However, since this is the defining equation of the Hubble law (Harrison 1993) and is often used in observational cosmology, it is useful to list it here alongside the previously discussed cosmological distances and adopt Eq. (6) as the defining expression of $d_{z}$ for all $z$.

\subsection{Radial (non-average) statistical tools}

Let us now call generically by $d_{0}$ some observationally defined distance. It can be any of the four distances $d_{L}, d_{\mathrm{A}}, d_{\mathrm{G}}, d_{z}$ defined so far. If $S_{0}$ is the area of the observed spherical shell of radius $d_{0}$, and $V_{0}$ the observed volume of radius $d_{0}$, we have that,

$S_{0}=4 \pi\left(d_{0}\right)^{2}$,

$V_{0}=\frac{4}{3} \pi\left(d_{0}\right)^{3}$.
Following J. Wertz, I define the differential density $\gamma\left(d_{0}\right)=\gamma_{0}$ at a certain distance $d_{0}$ as being given by the following expression (Wertz 1970, 1971; see also Ribeiro \& Miguelote 1998),

$\gamma_{0}=\frac{1}{S_{0}} \frac{\mathrm{d} N_{\mathrm{c}}}{\mathrm{d}\left(d_{0}\right)}$

where $N_{\mathrm{c}}$ is the cumulative radial number count from the origin of sources.

The integral differential density $\gamma^{*}\left(d_{0}\right)=\gamma_{0}^{*}$ is the integration of $\gamma_{0}$ over the observational volume $V_{0}$. Its equation yields

$\gamma_{0}^{*}=\frac{1}{V_{0}} \int_{V_{0}} \gamma\left(d_{0}\right) \mathrm{d} V_{0}=\frac{3}{\left(d_{0}\right)^{3}} \int_{0}^{d_{0}} x^{2} \gamma(x) \mathrm{d} x$.

The two definitions above look similar to the conditional densities $\Gamma$ and $\Gamma^{*}$ appearing in Pietronero (1987). However, it is important to point out the difference between the radial (not averaged) $\gamma$ and $\gamma^{*}$ statistics and the non-radial, but averaged, $\Gamma$ and $\Gamma^{*}$ statistics. The differential density $\gamma$ and its integral version measure how the density scales as a function of distance, whatever distance choice, whereas the conditional density $\Gamma$ is an average quantity, measuring the density from an occupied point and then averaged over all realizations of a given stochastic process. For the galaxy distribution what one does is to use a volume average to calculate the conditional density $\Gamma$ and the average conditional density $\Gamma^{*}$, which is an averaged integral of $\Gamma$ (Pietronero 1987; Coleman \& Pietronero 1992; Sylos-Labini et al. 1998; Pietronero 2003). Therefore, computing radial $(\gamma)$ and non-radial $(\Gamma)$ statistics are the result of different operations.

Therefore, here I follow Wertz's $(1970,1971)$ original contribution and will be approaching this problem from a regular (analytical) viewpoint, in addition to assuming that classical general relativity is a valid and appropriate tool for modelling the smoothed out large scale distribution of galaxies. This means that this paper deals with radial (non-average) $\gamma$ statistics derived from theory and attempts to see what the final results could suggest for the calculation of the non-radial (average) $\Gamma$ statistics obtained from galaxy catalogues ${ }^{6}$.

\subsection{Radial statistics of the Einstein-de Sitter cosmological model}

The EdS metric may be written as (from now on $c=G=1$ ),

$\mathrm{d} s^{2}=\mathrm{d} t^{2}-a^{2}(t)\left[\mathrm{d} r^{2}+r^{2}\left(\mathrm{~d} \theta^{2}+\sin ^{2} \theta \mathrm{d} \phi^{2}\right)\right]$.

The observational distances will then be given by the expressions below,

$d_{L}(z)=\frac{2}{H_{0}}(1+z-\sqrt{1+z})$

$d_{\mathrm{A}}(z)=\frac{2}{H_{0}} \frac{(1+z-\sqrt{1+z})}{(1+z)^{2}}$

$d_{\mathrm{G}}(z)=\frac{2}{H_{0}}\left(\frac{1+z-\sqrt{1+z}}{1+z}\right)$,

${ }^{6}$ I have not used Wertz's original symbol $\rho_{\mathrm{D}}$ for the differential density as it could be confusing in the present context. 
whereas the cumulative number count yields,

$N_{\mathrm{c}}(z)=\frac{4}{H_{0} M_{\mathrm{g}}}\left(\frac{1+z-\sqrt{1+z}}{1+z}\right)^{3}$.

where $M_{\mathrm{g}}$ is the average galactic rest mass $\left(\sim 10^{11} M_{\odot}\right)$.

Considering Eqs. (7) and (8) we can define four observational spherical shells and volumes as follows:

$S_{L}=\frac{16 \pi}{\left(H_{0}\right)^{2}}(1+z-\sqrt{1+z})^{2}$

$S_{\mathrm{A}}=\frac{16 \pi}{\left(H_{0}\right)^{2}} \frac{(1+z-\sqrt{1+z})^{2}}{(1+z)^{4}}$,

$S_{\mathrm{G}}=\frac{16 \pi}{\left(H_{0}\right)^{2}}\left(\frac{1+z-\sqrt{1+z}}{1+z}\right)^{2}$,

$S_{z}=\frac{4 \pi z^{2}}{\left(H_{0}\right)^{2}}$,

$V_{L}=\frac{32 \pi}{3\left(H_{0}\right)^{3}}(1+z-\sqrt{1+z})^{3}$

$V_{\mathrm{A}}=\frac{32 \pi}{3\left(H_{0}\right)^{3}} \frac{(1+z-\sqrt{1+z})^{3}}{(1+z)^{6}}$,

$V_{\mathrm{G}}=\frac{32 \pi}{3\left(H_{0}\right)^{3}}\left(\frac{1+z-\sqrt{1+z}}{1+z}\right)^{3}$,

$V_{z}=\frac{4 \pi z^{3}}{3\left(H_{0}\right)^{3}}$

It is convenient to express all quantities in terms of the redshift. Therefore, Eq. (9) should be rewritten as below,

$$
\begin{aligned}
\gamma_{0} & =\frac{\mathrm{d} N_{\mathrm{c}}}{\mathrm{d} z}\left[S_{0} \frac{\mathrm{d}}{\mathrm{d} z}\left(d_{0}\right)\right]^{-1} \\
& =\frac{6}{H_{0} M_{\mathrm{g}}} \frac{(1+z-\sqrt{1+z})^{2}}{(1+z)^{7 / 2}}\left[S_{0} \frac{\mathrm{d}}{\mathrm{d} z}\left(d_{0}\right)\right]^{-1} .
\end{aligned}
$$

We are now able to write the differential density in terms of the redshift for each observational distance that enters in its defining equation above. They yield,

$\gamma_{L}=\frac{\mu_{0}}{(2 \sqrt{1+z}-1)(1+z)^{3}}$,

$\gamma_{\mathrm{A}}=\frac{\mu_{0}(1+z)^{3}}{(3-2 \sqrt{1+z})}$,

$\gamma_{\mathrm{G}}=\mu_{0}$,

$\gamma_{z}=\frac{4 \mu_{0}(1+z-\sqrt{1+z})^{2}}{z^{2}(1+z)^{7 / 2}}$,

where,

$\mu_{0}=\frac{3\left(H_{0}\right)^{2}}{8 \pi M_{\mathrm{g}}}$.
Two conclusions can immediately be drawn from the equations above. Firstly, the differential density $\gamma_{\mathrm{G}}$, calculated by means of the galaxy area distance $d_{\mathrm{G}}$, is constant for all redshift. In other words, the use of such a distance makes the analysis completely insensitive to any change in the dust distribution of the EdS cosmology. In other words, using $d_{\mathrm{G}}$ we reach the conclusion that the dust distribution is homogeneous everywhere, even along the past null cone (or the lookback time). This is a built-in feature of the model and means that this quantity is of no use for probing the possible inhomogeneity of the observable Universe. Such a result was also reached by Ribeiro (2001b) by means of a somewhat different path. Secondly, it is clear from Eq. (26) that $\gamma_{\mathrm{A}}$ diverges at $z=1.25$, losing then its analytical usefulness for higher redshifts.

Finally, we are now in a position to calculate the integral differential density for each observational distance. If we rewrite Eq. (10) in terms of the redshift we get that,

$\gamma_{0}^{*}(z)=\frac{1}{V_{0}(z)} \int_{0}^{z} \gamma_{0}(z) \frac{\mathrm{d} V_{0}(z)}{\mathrm{d} z} \mathrm{~d} z$.

Using the expressions obtained above for volume and the differential density in each observational distance we obtain the following equations for the integral differential density,

$\gamma_{L}^{*}=\mu_{0}(1+z)^{-3}$,

$\gamma_{\mathrm{A}}^{*}=\mu_{0}(1+z)^{3}$,

$\gamma_{\mathrm{G}}^{*}=\mu_{0}$,

$\gamma_{z}^{*}=8 \mu_{0}\left[\frac{1+z-\sqrt{1+z}}{z(1+z)}\right]^{3}$.

It is useful to define the average numerical density $\langle n\rangle$ of a certain observational distance $d_{0}$ as being given by

$\left\langle n_{0}\right\rangle=N_{\mathrm{c}} / V_{0}$.

With the expressions obtained so far it is straightforward to show that the equations below hold:

$\left\langle n_{L}\right\rangle=\gamma_{L}^{*}$,

$\left\langle n_{\mathrm{A}}\right\rangle=\gamma_{\mathrm{A}}^{*}$,

$\left\langle n_{\mathrm{G}}\right\rangle=\gamma_{\mathrm{G}}^{*}$,

$\left\langle n_{z}\right\rangle=\gamma_{z}^{*}$.

All results above agree and expand both Ribeiro (1995, 2001b). The former, however, limited the analysis to the luminosity distance, while the latter concentrated on the behaviour of the average densities and their implications for the possible observational smoothness of the Universe. Equations (25) and (31) are equal to the ones appearing in Ribeiro (1995), with the difference that here they are a function of the redshift, whereas in Ribeiro (1995) they were written in terms of $d_{L}$.

Notice again that $\gamma_{\mathrm{G}}^{*}$ and, therefore, $\left\langle n_{\mathrm{G}}\right\rangle$ are constant, meaning that if one carries out consistency checks (see Sect. 2.5 above) between possible observable departures from homogeneous distribution, even at small scales $(z<0.1)$ one should not use the galaxy area distance $d_{\mathrm{G}}$. It would seem incorrect to do so because this distance renders constant its associated average density for all $z$. 


\subsection{Theoretical distances}

We should now relate the expressions obtained above with other theoretical distances which often appear in the literature. The proper volume is defined as follows,

$\mathrm{d} V_{\mathrm{PR}}=a^{3} r^{2} \mathrm{~d} r \sin \theta \mathrm{d} \theta \mathrm{d} \phi$,

and the comoving volume yields,

$\mathrm{d} V_{\mathrm{C}}=r^{2} \mathrm{~d} r \sin \theta \mathrm{d} \theta \mathrm{d} \phi$.

It is easy to show that in the EdS cosmology the area distance and the proper distance $d_{\mathrm{PR}}$ are given by the same expression (Ribeiro 1992b, 1995, 2001b; Ribeiro \& Stoeger 2003),

$d_{\mathrm{PR}}=a[t(r)] r=d_{\mathrm{A}}$,

and, therefore, have the same relationship to the redshift as given by Eq. (13) above.

As it is well known, the comoving distance $d_{\mathrm{C}}$ may be written as below,

$d_{\mathrm{C}}=r=\frac{d_{\mathrm{PR}}}{a}=\left(\frac{18}{H_{0}}\right)^{1 / 3}\left(\frac{1+z-\sqrt{1+z}}{1+z}\right)$,

since

$a=\left[\frac{4}{9\left(H_{0}\right)^{2}}\right]^{1 / 3} \frac{1}{1+z}$.

Therefore, comparing with Eq. (14) it follows that,

$d_{\mathrm{G}}=\left[\frac{4}{9\left(H_{0}\right)^{2}}\right]^{1 / 3} d_{\mathrm{C}}$.

Thus, the comoving distance, and, as a consequence, the comoving volume, are just the galaxy area distance times a constant factor, having then the same analytical behaviour with $z$ as any expression containing $d_{\mathrm{G}}$.

It is often the case in studies of galaxy statistics to use the famous Mattig formula to convert redshifts to distances. In the EdS cosmology it is easily proven that

$d_{\text {MATTIG }}=\frac{2}{H_{0}}\left(\frac{1+z-\sqrt{1+z}}{1+z}\right)=d_{\mathrm{G}}$.

So, the distance given by Mattig's formula is nothing more than the galaxy area distance.

The results obtained above show that the analysis presented in this paper does not need to refer to these often adopted theoretical distances, since by studying the behaviour of $d_{\mathrm{A}}$ and $d_{\mathrm{G}}$ we will have them taken into account.

\subsection{Reply to Célérier \& Thieberger (2001)}

In attempting to justify their different approach to the problem, Célérier \& Thieberger (2001; from now on CT01) have stated the following on page 452 of their paper: “... the luminosity distance is the observable quantity relevant for radially measured distances (...) But, when looking at a cross sectional area (...) perpendicular to the light ray and subtending a solid angle (...), the observer must consider the area distance." Then they obtain expressions for the radial differential density and volume element different from the ones appearing above. Those expressions were then used as the basis for their subsequent study and conclusions.

CT01 seem to have assumed that because the area distance $d_{\mathrm{A}}$ is defined by means of a cross sectional area $\mathrm{d} \sigma_{\mathrm{A}}$, which is measured at the source's rest frame, it is then the "correct" quantity to define the area of the observed spherical shell, with all other distances being, therefore, incorrect to use in this case. Moreover, they believe that $d_{L}$ is the "correct" distance for radial measure. Let us see why this reasoning is not correct.

Any observed distance, be it $d_{L}, d_{\mathrm{A}}, d_{\mathrm{G}}$ or $d z$, define an observable spherical shell and a radial measure for a given $z$. Because these distances are different, the shells will then have different radii and areas, but, nevertheless, they can be calculated from observations because these distances can be defined and observed. Even theoretically defined distances like the comoving and proper distances will also define spherical shells. The particular aspect about the area distance is that if, and only if, in our astronomical observations we, independently of a cosmological model, had also been able to observe and tabulate intrinsically measured dimensions such as $\mathrm{d} \sigma_{\mathrm{A}}$ or $\ell_{\mathrm{A}}$ (see Eqs. (2), (5) and Fig. 1), then the method to relate those intrinsically measured dimensions with the observed solid angle $\mathrm{d} \Omega_{\mathrm{A}}$, or the observed angular dimension $\alpha$, would be by means of equations which will result in measuring the area distance $d_{\mathrm{A}}$. This has nothing to do with the definition of spherical shells and measuring their areas or radius.

Similarly, if, independently of a cosmological model, we were able to measure the intrinsic luminosity $L$ of a cosmological source, then by measuring its observed flux $F$ we will be able to relate these two quantities and obtain a distance called luminosity distance. Such a procedure has nothing to do with a so-called "radial distance measure", but it is simply a method to obtain a specific distance by means of two different, but directly observable, quantities. Supernova cosmology is, of course, based exactly on this methodology and, due to that, it is able to actually verify a cosmological model rather than assume one (Perlmutter \& Schmidt 2003).

CT01 confuse two different distances when they wrote their differential density ${ }^{7}$. With the notation adopted in this paper CT01's Eq. (20) can be written as follows:

$$
\begin{aligned}
\gamma_{\mathrm{CT} 01} & =\frac{1}{S_{\mathrm{A}}} \frac{\mathrm{d} N_{\mathrm{c}}}{\mathrm{d}\left(d_{L}\right)}=\frac{\mathrm{d} N_{\mathrm{c}}}{\mathrm{d} z}\left[S_{\mathrm{A}} \frac{\mathrm{d}}{\mathrm{d} z}\left(d_{L}\right)\right]^{-1} \\
& =\frac{3\left(H_{0}\right)^{2}}{8 \pi M_{\mathrm{g}}} \frac{(1+z)}{(2 \sqrt{1+z}-1)}
\end{aligned}
$$

Notice how two entirely different methods for measuring the distance of a cosmological source are included in the same equation. They further defined an observed volume element as follows (CT01's Eq. (21)),

$\mathrm{d} V_{\text {СТ01 }}=S_{\mathrm{A}} \mathrm{d}\left(d_{L}\right)$.

\footnotetext{
${ }^{7}$ Both Ribeiro (1995) and CT01 calculated radial (non-average) $\gamma$ statistics, but named them with the symbol $\Gamma$ for non-radial (average) statistics. As discussed above (see Sect. 3.1) $\gamma$ and $\Gamma$ are different quantities and, therefore, one should use different symbols to avoid confusion.
} 
Everything else in their paper follows from these two equations. It is important to point out that as at the same redshift $d_{L} \neq d_{\mathrm{A}}$ with $d_{L}>d_{\mathrm{A}}$ (see Sect. 2.2 above), in both expressions above there will be a mismatch between $S_{\mathrm{A}}$ and $d_{L}$. So, for a given $z$ the volume element $\mathrm{d} V_{\text {Ст01 }}$ will have a spherical area $S_{\mathrm{A}}$ whose radius $d_{\mathrm{A}}$ is smaller than the distance $d_{L}$ where this spherical area is supposed to be located. It seems difficult to justify such a procedure.

As we shall see below, the results obtained by CT01 are different from Ribeiro's (1995) because densities defined with the area distance grow forever, inasmuch as $d_{\mathrm{A}}$ has the peculiar feature of vanishing at the big bang. However, the luminosity distance behaves in an opposite manner and goes to infinity at the big bang. Therefore, a density defined with $d_{L}$ vanishes at the big bang, while another density defined with $d_{\mathrm{A}}$ tends to infinity (see Ribeiro 2001b). By confusing these two distances CT01 smooth out the eventual decrease of density in their expression, as compared to Ribeiro's (1995), due to an incorrect algebraic manipulation.

\section{Analysis}

We are now in a position to discuss the results obtained in the previous section. Figure 3 shows graphs of the differential density $\gamma$ for all observational distances (proper and comoving distance behave as $d_{\mathrm{A}}$ and $d_{\mathrm{G}}$ respectively - see Sect. 3.3 above). The plots show very clearly the entirely different behaviour of $\gamma_{\mathrm{A}}$ on one hand, and $\gamma_{z}$ and $\gamma_{\mathrm{L}}$ on the other. Notice that $\gamma_{\mathrm{G}}$ is constant for all $z$ and that $\gamma_{\mathrm{A}}$ diverges at $z=1.25$. Figure 4 shows the integral differential density $\gamma^{*}$ for each observational distance. Notice again the entirely different behaviour one obtains if one uses $d_{\mathrm{G}}$ instead of $d_{z}$ or $d_{L}$.

The homogeneous behaviour in this case is given by the function obtained with the galaxy area distance $d_{\mathrm{G}}$, and the plots show quite clearly that deviations from homogeneity start to occur at $z=0.01$. At $z=0.1$ this deviation is meaningful. Notice that if one adopts the distance as given by Mattig's formula or the comoving distance one ends up with the same behaviour as given by $d_{\mathrm{G}}$, having therefore, no deviation from homogeneity, by construction.

These results suggest that one should not adopt these distances when trying to probe whether or not the galaxy redshift survey data show, or do not show, an eventual homogeneous pattern. In this respect, it is important to point out that many authors do adopt those apparently unsuitable distances when trying to ascertain a possible homogenization of the galaxy distribution. Among a few recent analysis, both Tikhonov et al. (2000) and Martínez et al. (2001) started with Mattig's equation, while Cappi et al. (1998) adopted the comoving distance. On the other hand Sylos-Labini et al. (1998), Joyce et al. (1999) and Pietronero et al. (2003) seemed to have used $d_{z}$ instead.

So, in view of the results presented here one may be compelled to ask how those results may affect the statistics of data stemming from galaxy redshift surveys.

One is faced with a fundamental difficulty if one tries to answer this question by means of the analysis presented above. The main problem stems from the fact that the data gathered from galaxy redshift surveys is presented in terms of non-radial

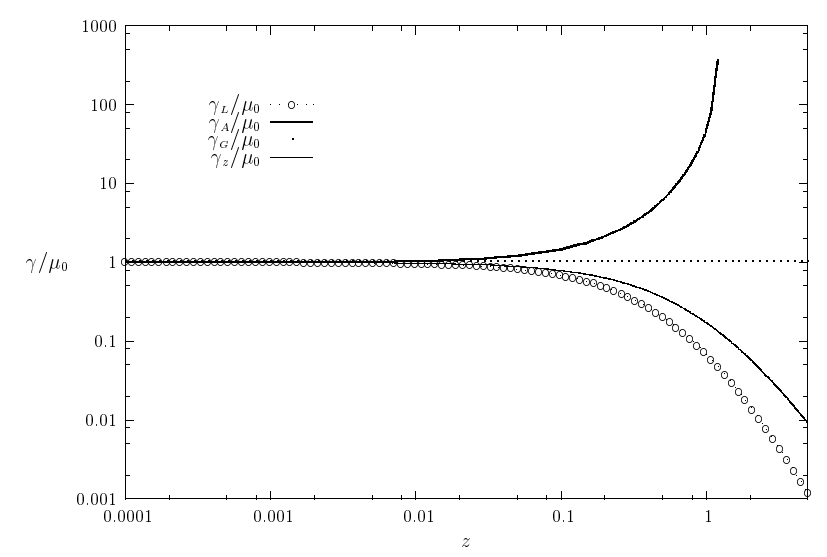

Fig. 3. Plot of the differential density $\gamma$ for each observational distance in EdS cosmology. Here $\mu_{0}$ is a constant factor related to the Hubble constant and galactic rest mass (see Eq. (29)). The proper and comoving distances are included, since the former is equal to $d_{\mathrm{A}}$, whereas the latter has the same behaviour against $z$ as $d_{\mathrm{G}}$. Notice that the distance obtained by means of Mattig's formula is equivalent to choosing $d_{\mathrm{G}}$ (see Sect. 3.3). The curves show very clearly the strong dependence of $\gamma$ with the chosen distance. $\gamma_{\mathrm{A}}$ diverges at $z=1.25$ and loses then its analytical significance for higher $z$. Notice too that by mixing up the opposite behaviour of $\gamma$ as given by $d_{\mathrm{A}}$ and $d_{L}$, CT01 have in fact smoothed out both the increase and decrease of $\gamma_{\mathrm{A}}$ and $\gamma_{L}$ respectively, explaining then the odd behaviour obtained by them.

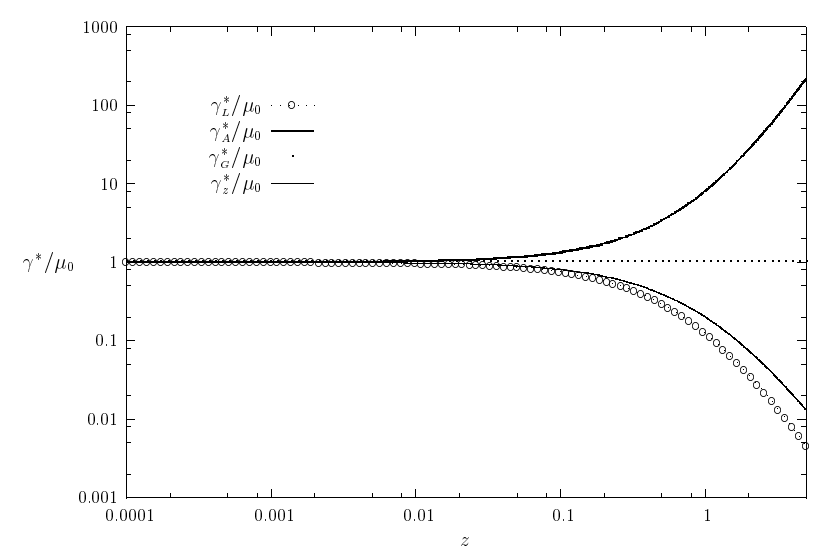

Fig. 4. Plot of the integral differential density $\gamma^{*}$ for each observational distance in EdS cosmological model ( $\mu_{0}$ is a constant factor defined in Eq. (29)). Both the comoving and the proper distances are included as they are respectively equivalent to $d_{\mathrm{G}}$ and $d_{\mathrm{A}}$. Again, the choice of distance fundamentally changes the behaviour of $\gamma^{*}$ for higher redshifts. The homogeneous situation is obtained when one adopts the galaxy area distance $d_{\mathrm{G}}$, or the comoving distance, or, still, the distance given by Mattig's formula (see Sect. 3.3). Notice that a significant deviation from the homogeneous case occurs at the relatively low value of $z=0.03(\approx 100 \mathrm{Mpc})$ when $\gamma_{\mathrm{G}}^{*}$ remains constant whereas all others do not. Since the integral differential density is the same as the average number density (see Eqs. (36)-(39)), $\gamma^{*}$ is the best statistical test for general tendencies of the galaxy clumping behaviour.

and averaged statistics, whereas here all quantities were discussed in terms of radial and non-averaged ones. As seen above, radial and non-radial statistics are the result of different operations (see Sect. 3.1). As clearly shown in Figs. 3 and 4 the use of different choices of distance produce distortions in 
the radial statistics and, thus, it is reasonable to suppose that similar distortions could affect the non-radial statistics as well. Nevertheless, there are no studies about this possible effect in statistics derived from galaxy catalogues as the distance problem has been mostly ignored so far in observational cosmology by both sides of the fractal controversy (Joyce et al. 1999, have, nonetheless, showed that non-radial densities are strongly affected by the kind of distance-redshift used). Therefore, the presence and strength of such a possible distortion showing itself in data obtained from galaxy catalogues remains, so far, an open problem.

Another important caveat is that the analysis presented above is bolometric, while real data analysis stemming from redshift surveys are always in a limited frequency range and often require the use of some form of K-correction. As discussed elsewhere (Ribeiro 2002; Ribeiro \& Stoeger 2003) a full relativistic analysis of radial number count where those effects are taken into consideration may affect very strongly the final quantities. In particular the luminosity function can only be compared with theoretical number count by means of complex equations which may seriously affect and change the theoretical quantities (Ribeiro \& Stoeger 2003). As noticed by Joyce et al. (1999), a simple luminosity function where those effects are not taken into account may change completely the final results of whether or not the data from the ESP galaxy survey show, or do not show, a scale invariant pattern. Other factors like the type of FLRW model as well as the existence or not of dark energy and a non-zero cosmological constant may also affect the statistics in unpredictable ways. Finally, even the calculation of the average density is affected by those effects (Ribeiro \& Stoeger 2003). It is beyond the scope of this paper to carry out a fully relativistic analysis to derive the average density from the luminosity function parameters, which themselves were calculated from galaxy catalogues, that is, where the data was obtained with limited frequency range observations, as well as some kind of morphological classification. Such an analysis should shed some light on those issues (Albani \& Ribeiro 2004, in preparation), but we may well assume in advance that those effects may be strong.

Considering those caveats the safest thing that could be said at this stage is that observers should take into consideration the possible distortion of their statistical analyses of galaxy surveys due to the adoption of different distance definitions. This may be especially true in the fractal controversy. Comparing data reduced with the comoving distance or by means of the Mattig formula with data reduced with the redshift distance could, perhaps, be one of the sources of controversy. Therefore, it would be very interesting to see the results if Cappi et al. (1998), Tikhonov et al. (2000) and Martínez et al. (2001) were to reduce their data by assuming either $d_{L}$ or $d_{z}$, and if Sylos-Labini et al. (1998) and Pietronero et al. (2003) were to do the same, but adopting now $d_{\mathrm{C}}$ or $d_{\mathrm{G}}$.

\section{Conclusion}

In this paper, I have discussed the effect of the distance choice in statistical tools used for possible fractal characterization of the galaxy distribution. I reviewed the basic notions of measuring distances of cosmological sources and used the area distance $d_{\mathrm{A}}$, the galaxy area distance $d_{\mathrm{G}}$, the luminosity distance $d_{L}$, the redshift distance $d_{z}$, as well as the comoving and proper distances, $d_{\mathrm{C}}$ and $d_{\mathrm{PR}}$ respectively, to calculate the differential density $\gamma$ and the integral differential density $\gamma^{*}$ of the dust distribution in a Einstein-de Sitter cosmology. The results showed the fundamental role played by the choice of distance in the determination of the scale where relativistic corrections must be taken into account as both $\gamma$ and $\gamma^{*}$ are strongly affected by the choice of distance. It is also shown that an inappropriate distance choice may lead to a failure to find evidence of a galaxy fractal structure when one calculates those two statistics, even if a self-similar structure does exist in the galaxy distribution. In particular, $d_{\mathrm{G}}, d_{\mathrm{C}}$ and the distance given by Mattig's formula seem unsuitable to probe for a fractal pattern as these two radial densities become constant for all redshifts when those distances are used in their calculation. This is a built-in feature of the EdS cosmological model. The analysis also suggests that if a fractal pattern really exists at ranges greater than $100 \mathrm{Mpc}$, it can only be detected at that scale by these statistics if they are calculated with either $d_{L}$ or $d_{z}$, as these two distance definitions are the only ones where, by theory, $\gamma$ and $\gamma^{*}$ can decrease at higher redshifts. I have also shown that Célérier \& Thieberger's (2001) critique of Ribeiro's (1995) earlier study, partially re-obtained here, is incorrect due to misconceptions regarding relativistic distance definitions, rendering their objections impaired.

Acknowledgements. Thanks go to M. A. H. MacCallum for discussions on cosmological distances. I am also grateful to the referee for very useful remarks which improved the paper. Partial support from FAPERJ and Instituto do Milênio CNPq 620053/2001-1 is acknowledged.

\section{References}

Abdalla, E., Afshordi, N., Khodjasteh, K., \& Mohayaee, R. 1999, A\&A, 345, 22

Baryshev, Y., \& Teerikorpi, P. 2002, Discovery of Cosmic Fractals (Singapore: World Scientific)

Basset, B. A., \& Kunz, M. 2004, Phys. Rev. D, 69, 101305

Bonnor, W. B. 1972, MNRAS, 159, 261

Cappi, A., Benoist, C., da Costa, L. N., \& Maurogordato, S. 1998, A\&A, 335, 779

Célérier, M.-N., \& Thieberger, R. 2001 (CT01), A\&A, 367, 449

Charlier, C. V. L. 1908, Ark. Mat. Astron. Fys., 4, 1

Charlier, C. V. L. 1922, Ark. Mat. Astron. Fys., 16, 1

Coleman, P. H., \& Pietronero, L. 1992, Phys. Rep., 1992, 213, 311

de Vaucouleurs, G. 1970, Science, 167, 1203

Ellis, G. F. R. 1971, General Relativity and Cosmology, Proc. of the International School of Physics "Enrico Fermi", ed. R. K. Sachs (New York: Academic Press), 104

Ellis, G. F. R., Nel, S. D., Stoeger, W. R., \& Whitman, A. P. 1985, Phys. Rep., 124, 315

Etherington, I. M. H. 1933, Phil. Mag., 15, 761

Fournier D'Albe, E. E. 1907, Two New Worlds, I The Infra World; II The Supra World (London: Longmans Green)

Grujić, P. V. 2001, Serbian Astron. J., No. 163, 21

Grujić, P. V. 2002, Serbian Astron. J., No. 165, 45

Haggerty, M. J., \& Wertz, J. R. 1972, MNRAS, 155, 495 
Harrison, E. 1993, ApJ, 403, 28

Hogg, D. W. 1999 [arXiv: astro-ph/9905116]

Joyce, M., Montuori, M., Sylos-Labini, F., \& Pietronero, L. 1999, A\&A, 344, 387

Kayser, R., Helbig, P., \& Schramm, T. 1997, A\&A, 318, 680

Li, W., \& Filippenko, A. V. 2003, IAU Coll., 192 [arXiv: astro-ph/0310529]

Mandelbrot, B. B. 1977, Fractals: Form, Chance and Dimension (New York: Freeman)

Mandelbrot, B. B. 1983, The Fractal Geometry of Nature (New York: Freeman)

Martínez, V. J., López-Martí, B., \& Pons-Bordería, M.-J. 2001, ApJ, $554, \mathrm{~L} 5$

Perlmutter, S., \& Schmidt, B. P. 2003, Supernova and Gamma Ray Bursts, K. Weiler (Berlin: Springer), in press [arXiv: astro-ph/0303428]

Pietronero, L. 1987, Physica A, 144, 257

Pietronero, L., \& Sylos-Labini, F. 2000, Proc. of the 7th Course of Astrofundamental Physics [arXiv: astro-ph/0002124]

Pietronero, L., Bottaccio, M., Montuori, M., \& Sylos Labini, F. 2003, Fractals, 11 (Supp.), 271 [arXiv: cond-mat/0111471]

Ribeiro, M. B. 1992a, ApJ, 388, 1

Ribeiro, M. B. 1992b, ApJ, 395, 29
Ribeiro, M. B. 1993, ApJ, 415, 469

Ribeiro, M. B. 1994, Deterministic Chaos in General Relativity, ed. D. Hobbil, A. Burd, \& A. Coley (New York: Plenum Press), 269

Ribeiro, M. B. 1995, ApJ, 441, 477

Ribeiro, M. B. 2001a, Fractals, 9, 237 [arXiv: gr-qc/9909093]

Ribeiro, M. B. 2001b, General Relativity and Gravitation, 33, 1699

Ribeiro, M. B. 2002, Observatory, 122, 201

Ribeiro, M. B., \& Miguelote, A. Y. 1998, Brazilian J. Phys., 28, 132

Ribeiro, M. B., \& Stoeger, W. R. 2003, ApJ, 592, 1

Schneider, P., Ehlers, J., \& Falco, E. E. 1992, Gravitational Lenses (Berlin: Springer)

Sylos-Labini, F., Montuori, M., \& Pietronero, L. 1998, Phys. Rep., 293, 61

Tikhonov, A. V., Makarov, D. I., \& Kopylov, A. I. 2000, Bulletin Special Astrophysical Observatory, 50, 39

Uzan, J.-P., Aghanim, N., \& Mellier, Y. 2004, preprint [arXiv: astro-ph/0405620]

Wertz, J. R. 1970, Newtonian Hierarchical Cosmology, Ph.D. Thesis, University of Texas at Austin

Wertz, J. R. 1971, ApJ, 164, 227

Wesson, P. S. 1978, Ap\&SS, 54, 489

Wesson, P. S. 1979, ApJ, 228, 647 